\title{
Relevanz von Feinpartikeln in Hydrologie und Wasserwirtschaft: Herkunft, Transport, Wirkung und Management in natürlichen und technischen Systemen
}

\author{
Andreas Kurtenbach · Joachim Sartor • Reinhard Bierl • Bettina Eisold • Markus Casper · Joachim Hansen • \\ Lucien Hoffmann · Jean-Paul Lickes · Paul Schosseler · Wolfhard Symader • André Weidenhaupt
}

Received: 21. September 2010/ Akzeptiert: 21. September 2010/Online veröffentlicht: 16. Oktober 2010

(C) Springer-Verlag 2010

Feinpartikel bedeuten für die Wasserwirtschaft und Hydrologie eine ständige Herausforderung, denn sie stören die Wassernutzung, sind aber untrennbar mit unseren Gewässern verbunden. Den Wissenschaftlern ist es bisher nicht gelungen, Verhalten und Eigenschaften von Feinpartikeln so gut zu verstehen, dass dieses Thema von der internationalen Forschungsagenda verschwinden könnte.

Über 60 Teilnehmer aus Universitäten, Fachhochschulen, Forschungseinrichtungen, Behörden, Umweltämtern, Firmen und Ingenieurbüros fanden sich daher am 8. Oktober $2009 \mathrm{zu}$ einem Fachseminar in Trier ein, in dem ein internationales Forschungsthema und regionale wasserwirt-

A. Kurtenbach $(\bowtie) \cdot$ R. Bierl $\cdot$ B. Eisold $\cdot$ W. Symader

Universität Trier, Fach Hydrologie,

54286 Trier, Deutschland

E-Mail: kurtenbach@uni-trier.de

J. Sartor

Fachhochschule Trier, Fachrichtung Bauingenieurwesen,

Trier, Deutschland

M. Casper

Universität Trier, Fach Physische Geographie,

Trier, Deutschland

J. Hansen

Universität Luxemburg,

Faculté des Sciences de la Technologie et de la Communication,

Luxemburg, Luxemburg

L. Hoffmann

Centre de Recherche Public - Gabriel Lippmann,

Belvaux, Luxemburg

A. Weidenhaupt $\cdot$ J.-P. Lickes

Administration de la Gestion de l'Eau,

Luxemburg, Luxemburg

P. Schosseler

Centre de Recherche Public - Henri Tudor,

Esch-sur-Alzette, Luxemburg schaftliche Probleme aufeinandertrafen. Es war das siebte Treffen der Seminarreihe „Regionale Wasserwirtschaft in Theorie und Praxis“, deren Veranstaltungen seit 2002 an wechselnden Standorten in Trier und Luxemburg durchgeführt werden.

In vertiefender Rück- und Zusammenschau des Fachseminars wurde die in dieser Ausgabe erscheinende Beitragsreihe „Relevanz von Feinpartikeln in Hydrologie und Wasserwirtschaft" entwickelt. Die Autoren dieses Editorials sind als Organisationskomitee der Tagung die mitverantwortlichen Herausgeber der einzelnen Beiträge.

Im Beitragsblock „Natürliche Systeme“ werden die Interaktionen von physikalischen, chemischen und biologischen Prozessen betont, welche die Feinpartikeldynamiken steuern. Der Analyse des Transportverhaltens und der Identifikation von Feinpartikelquellen und -senken kommt im Kontext eines einzugsgebietsweiten Sedimentmanagements besondere Bedeutung zu und wird zukünftig bei gewässerschutzrelevanten Maßnahmen in der europäischen Wasserrahmenrichtlinie (WRRL) an Einfluss gewinnen.

Kurtenbach et al. von der Universität Trier stellen in ihrem Beitrag die wichtigsten Erkenntnisse und Forschungsdefizite heraus. Durch kleinräumige Strukturen im Bachbett laufen Erosion und Sedimentationsprozesse gleichzeitig ab, was jedoch schwer zu modellieren ist. Ungeklärt sind die kurzzeitige Zwischenspeicherung und die Rolle des hyporheischen Interstitials sowohl für den Transport des Feinmaterials selbst als auch für den Schad- und Nährstofftransport und die gesamte ökologisch-toxikologische Bewertung.

Breitung und Keller von der Bundesanstalt für Gewässerkunde in Koblenz gehen das Problem des Baggergutes an. Abgelagerte Feinsedimente stören unter anderem den Schiffsverkehr und müssen beseitigt werden. Das allein ist schon aufwendig genug, aber wenn das Baggergut zusätzlich kontaminiert ist - eine typische Situation in Flusshä- 
fen - dann geht es nicht mehr um eine Massenverlagerung allein. Zusätzlich müssen neben dem Wasserrecht auch Fragen beantwortet werden, die aus dem Abfallrecht, dem Naturschutz oder dem Bodenschutz stammen. Beim Fallbeispiel Rhein müssen die Handlungsanleitungen überdies international abgesprochen werden.

Brinkmann et al. (Institut für Umweltforschung, RWTH Aachen), Cofalla et al. (Institut für Wasserbau und Wasserwirtschaft, RWTH Aachen) und Gerbersdorf (Institut für Wasserbau, Universität Stuttgart) schildern die Schwierigkeiten, die Umweltrisiken von Schwebstoffen und Feinsedimenten zu bewerten. Ihr Lösungsansatz geht aus einigen interdisziplinären Projekten hervor und ist multifaktoriell und integrativ.

Ursache-Wirkungs-Analysen sind die Grundlage jeder ernstzunehmenden Bewirtschaftungsmaßnahme innerhalb der WRRL. Doch was ist noch möglich, wenn die Ursachen nicht bekannt und die Wirkungsketten unklar sind? Gallé (Kompetenzzentrum technischer Umweltschutz - CRTE/CRP Henri Tudor, Luxemburg) bevorzugt bei Emissionsmodellen das Ereignis als Grundlage der Bewertung und mahnt die fehlende Verbindung zwischen chemischen und biologischen Parametern an. Die entscheidende „Blackbox“ aber ist der temporäre Feinmaterialspeicher „natürliches Flusssediment“.

Hohe Relevanz besitzen Feinpartikel aber nicht nur in natürlichen Gewässern. Auch in technischen Systemen sind mit auftretenden Partikeln hohe Anforderungen verbunden. So sind beispielsweise im Trinkwasser Inhaltsstoffe wie feinpartikuläre Trübstoffe, Eisen, Mangan und Bakterien unerwünscht und erfordern spezifische aufbereitungstechnische Maßnahmen. Darüber hinaus stellt die Beseitigung von gewässerschutzrelevanten Feinpartikeln in den Überund Abläufen von Regenrückhalteanlagen, Straßenentwässerungs- und Kanalisationssystemen sowie von Kläranlagen eine hohe Herausforderung dar, um beispielsweise FirstFlush-Phänomene und damit verbundene hohe Stoffbelastungen in den angrenzenden Gewässern zu vermeiden.

Die Aussagen von Fuchs et al. (Institut für Wasser und Gewässerentwicklung, Universität Karlsruhe) sind in diesem Zusammenhang präzise und eindeutig. Probleme be- reiten die Feinstoffe und nicht das Grobmaterial. Für einen Rückhalt werden Retentionsbodenfilter, Absetzanlagen mit einer ausreichenden Größenordnung $\left(>100 \mathrm{~m}^{3} / \mathrm{ha}\right.$ ) oder aber neue und effiziente Methoden wie der Einsatz von Schrägklärelementen oder gezielten Ablaufsteuerungen in der Regenabflussbehandlung benötigt.

Welker vom FG Siedlungswasserwirtschaft der FH Frankfurt stellt heraus, dass die abfiltrierbaren Stoffe hinsichtlich ihrer Schadstoffbelastung und Bedeutung für den Gewässerschutz erst in der jüngeren Vergangenheit diskutiert wurden. Die ständige Bewertungsfrage nach der Effizienz von Misch- und Trennkanalisationen wie auch die geringe Datenbasis insbesondere bei der Feststoffbelastung mit organischen Schadstoffen wird auch in Zukunft zu einem wasserwirtschaftlichen Dauerbrenner.

Schöpfer et al. (Björnsen Beratende Ingenieure $\mathrm{GmbH}$ ) stellen ihr Konzept für die Einhaltung eines Gewässergütestandards bei der Anlage eines künstlichen Sees vor. Neben den gelösten Stoffen sind es vor allem die Feinsedimente, die zurückgehalten werden müssen. Auch in diesem Fall liegen gute Erfahrungen mit Bodenfilteranlagen vor.

Lambert et al. (Stadtwerke Trier) lenken den Blickpunkt auf die Trinkwassergewinnung aus Oberflächengewässern. Hier ist die Partikelproblematik ein Teil des Reinigungsprozesses. Partikel werden eliminiert, entstehen aber auch während der Flockungsprozesse neu; und Partikel sind nicht immer anorganisch, sondern auch organisch-planktonisch. Die angestrebte Lösung ist die Ultrafiltration, und die Überwachung geschieht mithilfe eines Partikelzählers. Das ist in der Tat eine erhebliche Verbesserung gegenüber der traditionellen Trübungsmessung.

Die Beiträge zeigen somit ein multi- und interdisziplinäres Band über aktuelle Forschungsprojekte zur Schwebstoff- und Feinsedimentdynamik in natürlichen Gewässersystemen bis hin zur Relevanz von feinsuspendierten Feststoffen in technischen Systemen und diesbezüglichen Problematiken und Lösungsansätzen.

Wir danken dem Springer-Verlag und den Herausgebern für die Möglichkeit der Publikation der Fachbeiträge in dieser Zeitschrift. 